\title{
Cardiometabolic risk factors and lactoferrin: polymorphisms and plasma levels in French-Canadian children
}

\author{
Valérie Marcil ${ }^{2}$, Sylvain Mayeur ${ }^{1}$, Benoît Lamarche ${ }^{3}$, Jade England ${ }^{4}$, Mélanie Henderson ${ }^{4}$, Edgard Delvin ${ }^{1}$, Devendra Amre ${ }^{4}$ \\ and Emile Levy ${ }^{2}$
}

BACKGROUND: Lactoferrin (LTF) could play a beneficial role in insulin resistance and diabetes, but the association of its gene variants with cardio-metabolic disorders in children has not been investigated. This study aimed to examine the relationship between LTF variants, plasma LTF concentrations, and cardio-metabolic risk factors in French-Canadian children. METHODS: The study cohort comprises 1,749 French Canadians aged 9, 13, and 16 years. The association of 13 LTF polymorphisms, metabolic parameters, and plasma LTF levels was tested in this cross-sectional, province-wide schoolbased survey.

RESULTS: None of the genetic association remained significant after correction for multiple testing and LTF SNPS were not associated with LTF levels. Plasma LTF was positively correlated with body mass index $\left(r^{2}=0.2245, P=0.0011\right)$ and weight $\left(r^{2}=0.2515, P=0.0008\right)$. After segregating according to high-density lipoprotein cholesterol ( $\mathrm{HDL}-\mathrm{C})$, the association remained only in subjects exhibiting low HDL-C $\left(r^{2}=0.3868\right.$, $P=0.0002$ for body mass index and $r^{2}=0.3665, P=0.0004$ for weight). In girls, plasma LTF was positively correlated with total cholesterol $\left(r^{2}=0.2231, P=0.0378\right)$, LDL cholesterol $\left(r^{2}=0.2409, P=0.0246\right)$, and apolipoprotein $B\left(r^{2}=0.2478\right.$, $P=0.0207)$.

CONCLUSIONS: We found no association between LTF gene variants and metabolic parameters following correction for multiple testing. HDL-C and gender-specific positive associations were evidenced between plasma LTF, anthropometric profile, and lipid levels.

$T$ he disconcerting rise in the rates of obesity in children and adolescents has contributed to increasing their risk for developing insulin resistance and other cardio-metabolic complications such as dyslipidemia. Insulin resistance/glucose intolerance, abdominal obesity, elevated blood pressure, and dyslipidemia are components of the metabolic syndrome (MetS), a cluster of medical disorders that enhance the risk of developing type 2 diabetes (T2D) and cardiovascular diseases (1). The development of T2D in young people is thus of particular concern because several complications are common and appear early in the disease (2). Consequently, the identification of primary markers and genetic risk factors for insulin resistance and $\mathrm{T} 2 \mathrm{D}$ in young children are becoming important tools for the management and the prevention of long-term cardio-metabolic consequences.

Lactoferrin (lactotransferrin, $L T F$ ) is an $80-\mathrm{kDa}$, monomeric multifunctional iron-binding glycoprotein produced by neutrophils and by epithelial glands, and is present in different cells and body fluids. LTF receptors exist in a variety of cells, including adipocytes, hepatocytes, endothelial cells, monocytes, and lymphocytes (3). It has been reported that LTF displays antibacterial (4), antiviral (5), anti-inflammatory (6), and antioxidant (7) actions.

LTF may exert beneficial effects on metabolic parameters. In rodents, oral administration of bovine LTF led to decreased plasma triacylglycerol (TG) and free fatty acid (FFA) levels, to increased plasma high-density lipoprotein cholesterol (HDL-C) concentrations, and to low hepatic cholesterol and TG content (8). Bovine LTF administration to mice also reduced mesenteric fat tissue and hepatic TG (9). In a macrophage cellular model, LTF inhibited the uptake of acetylated (10) and oxidized low-density lipoprotein (LDL) (11). In humans, circulating LTF levels were found to be lower in obese subjects, and were inversely associated with fasting TG, body mass index (BMI), and plasma fasting glucose, while being directly correlated with HDL-C levels (12). Moreover, in severely obese patients, circulating LTF concentrations were inversely associated with postprandial lipemia, parameters of oxidative stress (e.g., catalase and glutathione peroxidase), and with C-reactive protein, both at baseline and after a fat overload (13). Conversely, in non-diabetic Latino adolescents, plasma LTF was positively associated with BMI, fat mass, and LDL-C levels (14). Therefore, inconsistency among studies support the need to further explore the association between LTF levels, anthropometric profile, and metabolic parameters.

Several polymorphisms in the LTF gene have been studied for their correlation with circulating LTF levels (15), biochemical parameters (12), or risks for pathological conditions

\footnotetext{
${ }^{1}$ Research Centre, CHU Sainte-Justine, Université de Montréal, Montreal, Quebec, Canada; ${ }^{2}$ Department of Nutrition, Université de Montréal, Montreal, Quebec, Canada; ${ }^{3}$ Institute of Nutrition and Functional Foods (INAF), Laval University, Quebec City, Quebec, Canada; ${ }^{4}$ Department of Pediatrics, Université de Montréal, Montreal, Quebec, Canada. Correspondence: Emile Levy, E-mail: (emile.levy@recherche-ste-Justine.qc.ca)

Received 22 December 2016; accepted 11 February 2017; advance online publication 16 August 2017. doi:10.1038/pr.2017.72
} 


\section{Articles $\mid$ Marcil et al.}

such as susceptibility to diarrhea (16), Parkinson disease (17), and coronary artery stenosis (15). Associations with lipid levels were revealed in adult obese populations (12), but such investigations have not been performed in children. The aim of this study was to investigate the relationship between LTF genetic variants and the presence of cardio-metabolic risk factors in a pediatric French Canadian population. Another goal was to explore the association between LTF plasma levels and cardio-metabolic parameters in this pediatric cohort.

\section{METHODS}

\section{Population}

The design and methods of the 1999 Quebec Child and Adolescent Health and Social Survey (QCAHSS), a school-based survey of youth aged 9,13 , and 16 years, have previously been reported in detail (18). On a total of 2,244 DNA samples available, we restricted the current analysis only to the 1,749 unrelated children and adolescents of French Canadian origin in order to reduce the confounding of genetic analyses by population stratification. The Institutional Review Board of Sainte-Justine Hospital approved the study and investigations were carried out in accordance with the principles of the Declaration of Helsinki. Written informed consent was obtained from parents/guardians, and written informed assent was obtained from study participants.

\section{Anthropometry, Blood Pressure, and Lipids}

Height, weight, and blood pressure (BP) were measured according to the standardized protocols (18). BMI was computed as weight in kilograms divided by the height in meters squared $\left(\mathrm{kg} / \mathrm{m}^{2}\right)$. Values of percentile cutoff points used to identify subjects with metabolic risk factors were estimated from the study distributions. Cutoff points were age and sex specific, and BP cutoff points were also height specific, according to the National High Blood Pressure Education Program Working Group on High Blood Pressure in Children and Adolescents. Subjects with BMI $\geq 85$ th percentile values were categorized as overweight/obese. High TG, insulin, LDL-C, and systolic BP were defined as values $\geq 75$ th percentile, and low HDL-C was defined as values $\leq 25$ th percentile. Impaired fasting glucose was defined as concentrations $\geq 6.1$ and $<7.0 \mathrm{mmol} / \mathrm{l}$. No study participant had fasting plasma glucose $\geq 7.0 \mathrm{mmol} / \mathrm{l}$. Estimation of childhood MetS prevalence continues to be challenging and controversial and there is no internationally accepted definition of childhood MetS. More than 40 definitions for childhood MetS have so far been proposed and most of them are based on adaptations of adult criteria. Therefore, in the present work, we have based our definition on our work where we assessed the clustering of metabolic risk factors and estimated the prevalence of MetS in a representative sample of youth in the province of Quebec in Canada (19). In our analyses, MetS required the presence of obesity and at least two other risk factors among high systolic or diastolic BP, high TG, low HDL$\mathrm{C}$, and impaired fasting glucose (1). General obesity was used instead of central obesity, as waist circumference data were not available for this study.

\section{Biochemical Analyses}

Blood samples were collected in the morning, after an overnight fast. Plasma total cholesterol (TC), HDL-C, TG, and glucose concentrations were determined on a Beckman Synchron Cx7 instrument as previously described (18). Apolipoprotein (apo) A-I and apo B were measured by nephelometry (Array Protein System; Beckman). The Friedewald equation was used to calculate LDL-C. Plasma insulin concentrations were determined with the ultrasensitive Access immunoassay system (Beckman Coulter, Brea, CA, USA), which has no cross-reactivity with proinsulin or C-peptide. Plasma FFA concentrations were quantified by an enzymatic colorimetric method (Wako Chemicals, Richmond, VA, USA). Plasma LTF was measured using a commercial ELISA kit (AssayPro, St Charles, MO) in a sample of 176 children aged 9, 13, and 16 years, selected according to their extreme HDL-C values in order to detect the largest effect.

\section{Genotyping}

Genomic DNA was prepared from white blood cells using the Puregene DNA Isolation kit (Gentra Systems, Minneapolis, MN, USA). In total, 13 LTF SNPs were selected based on previous associations reported in the literature (12,15-17,20-25) (Supplementary Table S1 online). SNPs were genotyped using Sequenom-based primer-extension methods. These methods are designed for high-throughput SNP genotyping. The platform has a high assay conversion rate (85\%), high genotyping success rate $(95 \%)$, and minimal error rates $(0.5-1 \%)$. Genotyping was carried out at the McGill University and Genome Quebec Innovation Center in Montreal.

\section{Statistical Analysis}

Statistical analyses were performed with STATA v.10 statistical software (StataCorp LP, College Station, TX, USA). Potential genotyping errors were assessed using $\chi^{2}$ tests, which evaluate the deviation of each SNP from Hardy-Weinberg equilibrium. Subjects were categorized according to their MetS status (yes/no). Betweengroup allele and genotype frequency distributions were compared by a $\chi^{2}$ test. Allelic association for individual SNPs was carried out using logistic regression by fitting an additive model. To take the design effect into account, mixed models were used for all analyses of variance and regressions, with genetic markers and other independent variables treated as fixed effects, and with clustering between subjects in the same school treated as a random effect. We used mixed logistic regression to examine the association between MetS status and LTF genotypes. We used mixed analysis of variance and mixed linear regression to study the associations between genotypes and metabolic variables. Scheffe's contrasts were used for post hoc pair comparisons. Insulin, TG, FFA, and BMI values were logtransformed for statistical analyses to improve the normality of their distributions. Because we pooled age and sex groups, age- and sexspecific $Z$-scores for BMI, insulin, glucose, TG, LDL-C, HDL-C, apo $\mathrm{B}$, and apo A-I were used in linear regression analyses. To standardize a value (i.e., compute its $Z$-score), we subtracted the mean of the corresponding study distribution and divided it by the SD. Haplotype analysis was carried out using HAPLOVIEW Software (Broad Institute, Cambridge, MA, USA), version 3.11 on the six SNPs for which the allelic association was significant or close to significant. Haplotype blocks were created using the confidence interval feature. For each block, the haplotype association for each haplotype with MetS was examined by logistic regression. The association with the metabolic markers was evaluated using linear regression and $P$ values were estimated. Pearson's correlation analyses were used to estimate correlation between LTF levels and various phenotypes (confidence interval of 95\%).

\section{RESULTS}

\section{Population Characteristics}

The clinical and biochemical characteristics of participants are shown in Table 1. The prevalence of MetS was $11.03 \%$. As expected, youth with MetS displayed significantly higher BMI, systolic and diastolic BP, TC, LDL-C, apo B, TG, FFA, insulin, and glucose, as well as lower levels of HDL-C and apo A-I than youth without MetS. No differences were detected in age and gender among subjects with and without MetS.

\section{Effect of Polymorphisms on Metabolic Variables}

A total of 1,749 subjects were included for genotyping. The list of the 13 genotyped SNPs, their associations reported in the literature, and their minor allele frequencies are illus- 
Table 1. Characteristics of study participants according to metabolic syndrome status

\begin{tabular}{|c|c|c|c|c|}
\hline \multirow[t]{2}{*}{ Variable } & \multirow[t]{2}{*}{ Total $(n=1,749)$} & \multicolumn{2}{|c|}{$\mathrm{MetS}^{\mathrm{a}}$} & \multirow[t]{2}{*}{$P$ value $^{\mathrm{b}}$} \\
\hline & & No $(n=1,556)$ & Yes $(n=193)$ & \\
\hline 9-Year olds, \% (n) & 31.96 (559) & $32.33(503)$ & $29.02(56)$ & 0.648 \\
\hline 13-Year olds, \% (n) & $30.87(540)$ & $30.72(478)$ & $32.12(62)$ & - \\
\hline 16 -Year olds, \% (n) & $37.16(650)$ & 36.95 (575) & $38.86(75)$ & - \\
\hline Gender: male, \% ( $n)$ & $50.31(880)$ & $50.39(784)$ & $49.74(96)$ & 0.866 \\
\hline $\mathrm{BMI}^{\mathrm{c}}\left(\mathrm{kg} / \mathrm{m}^{2}\right)$ & $20.23 \pm 4.37$ & $19.30 \pm 3.28$ & $28.01 \pm 4.55$ & $<0.00001$ \\
\hline Systolic BP (mm Hg) & $111.88 \pm 13.74$ & $110.64 \pm 13.00$ & $122.01 \pm 15.32$ & $<0.00001$ \\
\hline Diastolic BP (mm Hg) & $59.34 \pm 7.14$ & $58.79 \pm 6.95$ & $63.77 \pm 7.09$ & $<0.00001$ \\
\hline $\mathrm{TC}(\mathrm{mmol} / \mathrm{l})$ & $4.00 \pm 0.75$ & $3.97 \pm 0.75$ & $4.20 \pm 0.80$ & $<0.00001$ \\
\hline LDL-C (mmol/l) & $2.31 \pm 0.64$ & $2.28 \pm 0.63$ & $2.48 \pm 0.67$ & $<0.00001$ \\
\hline Apo B (g/l) & $0.66 \pm 0.18$ & $0.65 \pm 0.17$ & $0.75 \pm 0.20$ & $<0.00001$ \\
\hline $\mathrm{HDL}-\mathrm{C}(\mathrm{mmol} / \mathrm{l})$ & $1.30 \pm 0.25$ & $1.32 \pm 0.25$ & $1.13 \pm 0.18$ & $<0.00001$ \\
\hline Apo A-I (g/l) & $1.19 \pm 0.17$ & $1.20 \pm 0.17$ & $1.13 \pm 0.16$ & $<0.00001$ \\
\hline $\mathrm{TG}^{\mathrm{C}}(\mathrm{mmol} / \mathrm{l})$ & $0.87 \pm 0.42$ & $0.82 \pm 0.36$ & $1.28 \pm 0.63$ & $<0.00001$ \\
\hline $\mathrm{FFA}^{\mathrm{c}}(\mathrm{mmol} / \mathrm{l})$ & $0.44 \pm 0.21$ & $0.43 \pm 0.21$ & $0.47 \pm 0.20$ & $<0.0086$ \\
\hline Glucose (mmol/l) & $5.16 \pm 0.38$ & $5.15 \pm 0.38$ & $5.26 \pm 0.40$ & $<0.0001$ \\
\hline Insulin ${ }^{\mathrm{c}}$ (pmol/l) & $43.62 \pm 30.50$ & $38.71 \pm 20.20$ & $83.23 \pm 58.24$ & $<0.00001$ \\
\hline \multicolumn{5}{|c|}{ 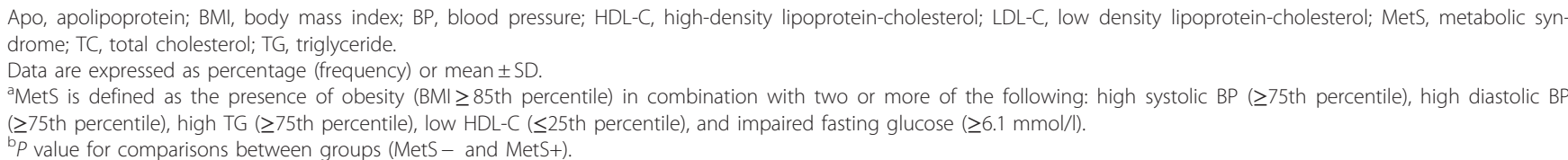 } \\
\hline
\end{tabular}

Table 2. LTF polymorphisms and metabolic parameters

\begin{tabular}{|c|c|c|c|c|c|}
\hline SNP & Metabolic parameter & Alleles (major/minor) & Adjusted coefficient & $P$ value & Corrected $P$ value \\
\hline rs1126478 & $\mathrm{HDL}-\mathrm{C}(\mathrm{mmol} / \mathrm{l})$ & $A / G$ & $0.086 \pm 0.035$ & 0.015 & 0.195 \\
\hline rs34827868 & $\mathrm{HDL}-\mathrm{C}(\mathrm{mmol} / \mathrm{l})$ & $\mathrm{C} / \mathrm{T}$ & $0.174 \pm 0.074$ & 0.018 & 0.234 \\
\hline rs1042073 & $\mathrm{HDL}-\mathrm{C}(\mathrm{mmol} / \mathrm{l})$ & $\mathrm{C} / \mathrm{T}$ & $0.089 \pm 0.036$ & 0.015 & 0.195 \\
\hline rs4637321 & Fasting glucose (mmol/l) & $\mathrm{C} / \mathrm{T}$ & $-0.076 \pm 0.039$ & 0.05 & 0.650 \\
\hline rs2239692 & Blood pressure $(\mathrm{mmHg})$ & $A / G$ & $1.314 \pm 0.163$ & 0.029 & 0.377 \\
\hline rs10865941 & $\mathrm{FFA}(\mathrm{mmol} / \mathrm{l})$ & $\mathrm{T} / \mathrm{C}$ & $0.074 \pm 0.034$ & 0.033 & 0.429 \\
\hline SNP & Effect of minor allele & & Odds ratio $(95 \% \mathrm{Cl})$ & $P$ value & Corrected $\mathrm{P}$ value \\
\hline rs2239692 & $\downarrow$ risk MetS & $A / G$ & $0.82 \pm 0.10(0.65-1.02)$ & 0.039 & 0.507 \\
\hline
\end{tabular}

FFA, free fatty acids; HDL-C, high-density lipoprotein cholesterol; MetS, metabolic syndrome.

A positive coefficient suggests increasing value of the marker for every additional copy of the SNP. A negative coefficient suggests decreasing value of the marker for every additional copy of the SNP. The linear mixed model was adjusted for age, gender and body mass index. Separate logistic regression models were fit for each SNP adjusting for age, gender, and body mass index.

For the risk of metabolic syndrome, separate logistic regression models were fit for each SNP adjusting for age, gender, and body mass index.

trated in Supplementary Table S1. All SNPs were in Hardy-Weinberg equilibrium. We studied the effect of $L T F$ polymorphisms on mean LDL-C, HDL-C, apo A-I, apo B, FFA, TG, glucose, and insulin levels. As we did not detect heterogeneity in $L T F$ polymorphism effects with respect to sex and age, groups were pooled in subsequent analyses. Regression coefficients were calculated to study associations between LTF genotypes and $Z$-score for blood glucose, insulin, TG, HDL-C, LDL-C, apo A-I, and apo B after adjustment for age, sex, and BMI. Three SNPs (rs1126478, rs34827868, and rs1042073) had minor allele associated with increased levels of HDL-C (coefficients: $0.086 \pm 0.035$, $P=0.015 ; \quad 0.174 \pm 0.074, \quad P=0.018 ; \quad$ and $\quad 0.089 \pm 0.036$, $P=0.015$, respectively; Table 2). A negative coefficient suggests decreasing values of the marker for every additional copy of the risk allele. Three other SNPs (rs4637321, rs2239692, and rs10865941) were associated respectively with reduced fasting glucose (coefficient: $-0.076 \pm 0.039$, 


\section{Articles | Marcil et al.}

$P=0.05)$, increased BP (coefficient: $1.314 \pm 0.163, P=0.029$ ), and elevated FFA (coefficient: $0.074 \pm 0.034, P=0.033$ ). However, none of these associations remained significant after correction for multiple testing.

Effect of Polymorphisms on the Risk of Metabolic Syndrome The 13 SNPs were analyzed for association with MetS and the results are presented in Table 2. Before correction for multiple testing, there was a significant difference in allele frequencies between $\mathrm{MetS}$ - and MetS+ subjects for one polymorphism (rs2239692, odds ratio (OR): $0.82 \pm 0.10$, $P=0.039$ ) that was found associated with blood pressure values. However, after correction for multiple comparisons, the association lost its significance.

\section{Haplotype Analyses}

Haplotype analyses were performed on the six LTF SNPs for which allelic associations were detected (Table 3). We found that one haplotype (frequency of $5.1 \%$ ) was associated with
HDL-C levels (coefficient: $0.195 \pm 0.077, P=0.011$ ) after adjustment for age, sex, and BMI, but the association lost its significance after correction for multiple testing.

\section{Effect of Polymorphisms on Plasma LTF Levels}

We studied the effect of the six LTF polymorphisms, for which allelic associations were detected, on plasma LTF levels in our sub-cohort of 176 subjects and did not find any effect even after correcting for age, sex, BMI, and HDL levels (Supplementary Table S2).

\section{Plasma LTF According to HDL Levels}

As we observed nominally significant $P$ values for multiple LTF SNPs with plasma HDL-C, we considered that this association was worth further investigation. Thus, we attempted to compare plasma LTF levels in subjects presenting with low and high plasma HDL-C. From our original cohort, we selected 176 subjects from both genders in three age categories (Supplementary Table S2). Data showed that, compared

Table 3. LTF haplotypes and plasma HDL-C levels

\begin{tabular}{llccccc}
\hline & Haplotype & Frequency (\%) & Adjusted coefficient & $95 \%$ Cl & $P$ value & Corrected $P$ value \\
\hline rs1042073, rs2239692, rs34827868, & GTAGAC & 5.1 & $0.195 \pm 0.077$ & 0.044 to -0.346 & 0.011 & 0.143 \\
rs1126478, rs4637321, rs10865941 & ACGAGC & 2.3 & $-0.093 \pm 0.048$ & -0.187 to 0.000 & 0.050 & 0.650
\end{tabular}

A positive coefficient suggests increasing value of the marker for every additional copy of the haplotype. A negative coefficient suggests decreasing value of the marker for every additional copy of the haplotype. The linear mixed model was adjusted for age, gender, and body mass index.

Table 4. Correlations between plasma lactoferrin levels and metabolic parameters

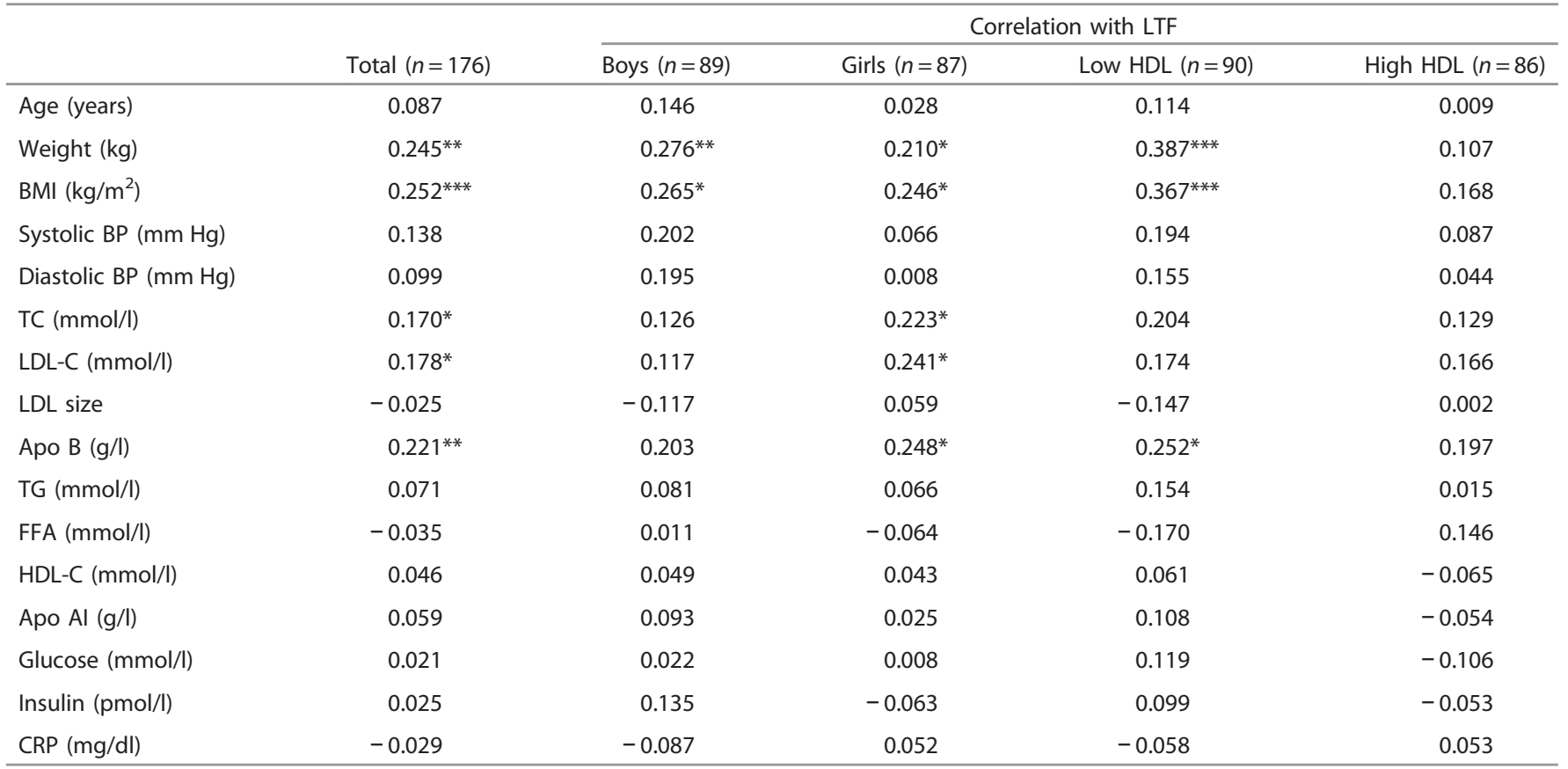

Apo, apolipoprotein; BMI, body mass index; BP, blood pressure; CRP, C-reactive protein; FFA, free fatty acids; HDL-C, high-density lipoprotein cholesterol; LDL-C, low-density lipoprotein cholesterol; MetS, metabolic syndrome; TC, total cholesterol; TG, triglyceride.

${ }^{*} P<0.05$; ${ }^{* *} P<0.01$; ${ }^{* * *} P<0.001$. 
to subjects with low HDL-C, subjects presenting with high HDL-C also had higher levels of apo A-I $(1.83 \pm 0.19$ vs. $0.86 \pm 0.08 \mathrm{~g} / \mathrm{l}, P<0.0001)$, total cholesterol $(4.48 \pm 0.61$ vs. $3.45 \pm 0.78 \mathrm{mmol} / \mathrm{l}, P<0.0001)$, and LDL-C (2.34 \pm 0.54 vs. $2.08 \pm 0.66 \mathrm{mmol} / \mathrm{l}, P=0.0042)$. However, they had lower BMI $\left(18.37 \pm 3.09\right.$ vs. $\left.21.45 \pm 5.28 \mathrm{~kg} / \mathrm{m}^{2}, P<0.0001\right)$, TG $(0.67 \pm$ 0.25 vs. $1.11 \pm 0.57 \mathrm{mmol} / \mathrm{l}, P<0.0001)$, insulin $(35.64 \pm 16.80$ vs. $49.31 \pm 27.28 \mathrm{pmol} / \mathrm{l}, P<0.0001)$, and C-reactive protein $(1.15 \pm 5.16$ vs. $3.92 \pm 8.90 \mathrm{mg} / \mathrm{dl}, P<0.0129)$. Nevertheless, no differences were observed in plasma LTF levels between the two groups (Supplementary Table S3).

\section{Associations between Plasma Lactoferrin and Metabolic Parameters}

Finally, we evaluated the associations between plasma LTF levels and metabolic parameters (Table 4). Our data show that plasma LTF is positively correlated with BMI and weight (Figure 1a). However, after segregating subjects according to their HDL-C status, the association was detectable only in subjects displaying low HDL-C (Figure 1b), as no correlation with weight or BMI was found in children with high HDL-C levels (Figure 1c). Moreover, plasma LTF was positively correlated with total cholesterol, LDL-C, and apo B levels in children (Figure 2a). Analyzing the data according to gender revealed significant associations only in girls (Figure $\mathbf{2 b}, \mathbf{c}$ ). Plasma glucose, insulin, C-reactive protein, and HDL-C were not significantly associated with LTF levels.

\section{DISCUSSION}

In this work, we have demonstrated that: (i) after correction for multiple testing, LTF polymorphisms and haplotypes are not associated with metabolic parameters in French-Canadian
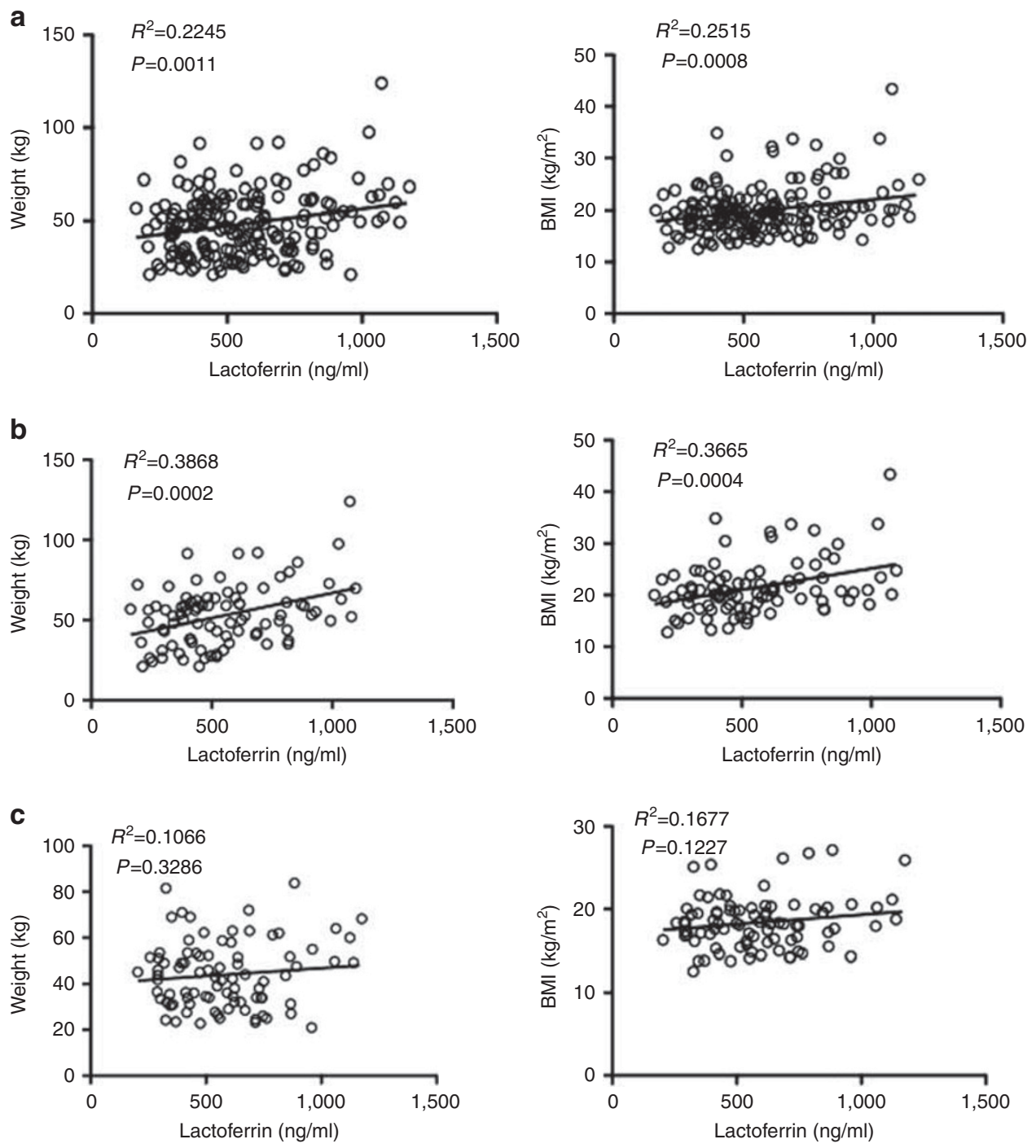

Figure 1. Correlations between plasma lactoferrin, weight and body mass index. Plasma levels of lactoferrin were measured and correlated in (a) all children ( $n=176)$; (b) children with low levels of HDL-C $(n=90)$ and; (c) with high levels of HDL-C $(n=86)$. 

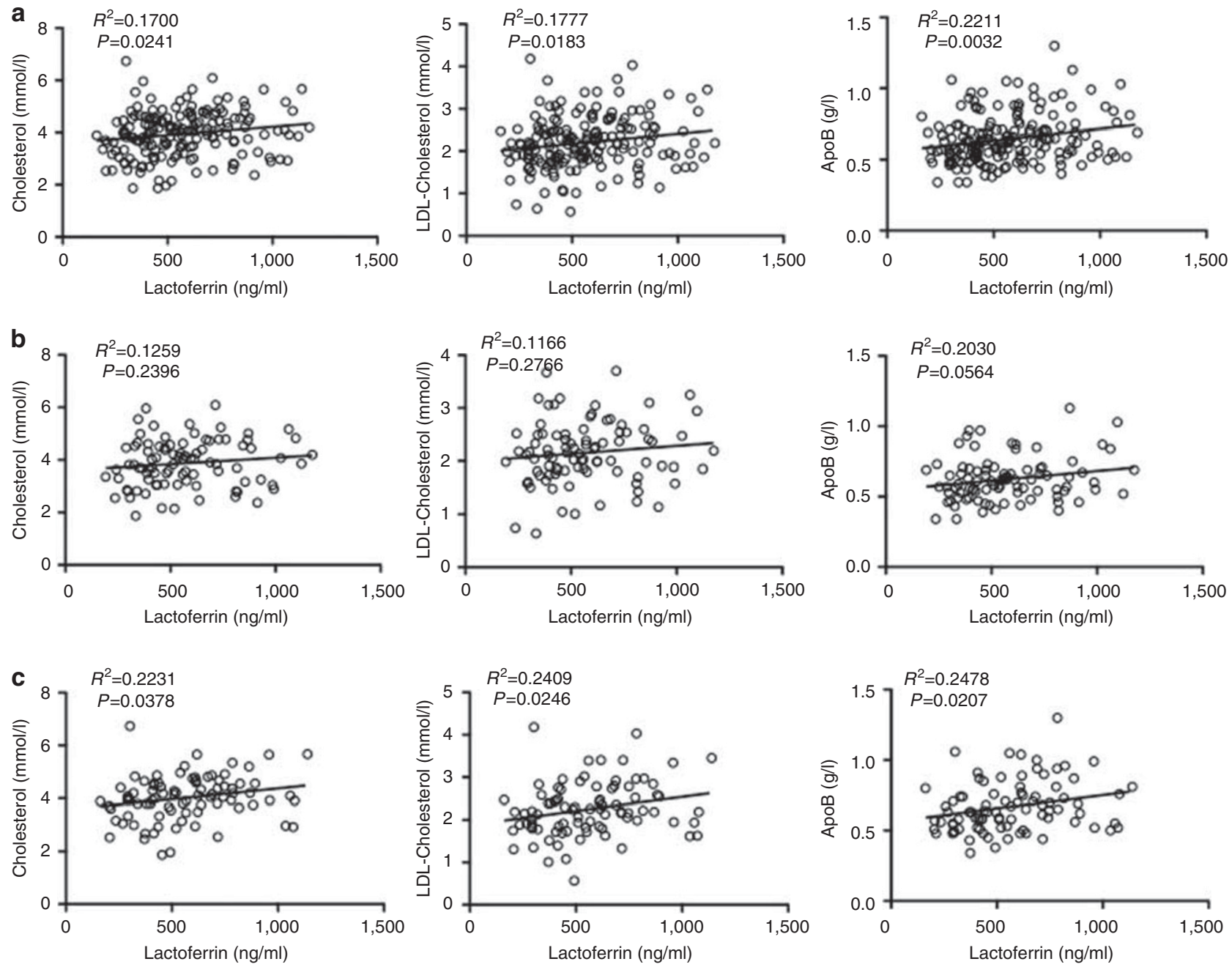

Figure 2. Correlations between plasma lactoferrin, total cholesterol, LDL-C and Apo B. Plasma levels of lactoferrin were measured and correlated in (a) all children ( $n=176)$; (b) boys $(n=89)$ and; (c) girls $(n=87)$.

children; (ii) LTF polymorphisms and haplotypes are not related to LTF plasma levels; and (iii) LTF plasma levels are correlated with metabolic outcomes in a HDL-C- and genderdependant manner.

Despite the large number of subjects included in our study, our results did not validate the associations previously reported by Moreno-Navarrete et al. (12). The authors found that, in men presenting with altered glucose tolerance, two non-synonymous polymorphisms (rs1126477 and rs1126478) were associated with TG and HDL-C plasma levels (12). In our study, the associations found with metabolic variables (HDL-C, fasting glucose, blood pressure, and FFA) or with the presence of MetS did not remain significant after corrections for multiple testing. In the same manner, after dosing plasma LTF in a sub-cohort of 176 subjects, we could not reveal associations with LTF polymorphisms, a finding that is in line with the results published by Moreno-Navarrete et al. (12). Conversely, in a cohort of 155 women and 150 men, Videm et al. (15) did show associations between the deletion variant of rs10662431 and the G allele of rs1126478 and higher plasma LTF concentrations.

Although no significant association was found, our study highlighted the tendency for association with plasma HDL-C. To further explore this issue, we measured plasma LTF in children presenting with low and high HDL-C levels. No differences in plasma LTF were found between the two groups, which is in accordance with a previous study showing that circulating LTF is not significantly associated with HDL$\mathrm{C}$ in obese subjects (13). Next, our analyses revealed a significant association between plasma LTF, weight, and BMI in children. Similarly, a study performed in non-diabetic Latino youth (163 subjects aged 8-21 years) revealed higher plasma LTF levels in overweight and obese subjects compared to those with normal weight (14). Combined with our results, these data demonstrate that the association between plasma LTF and BMI in children is different from that detected in adult populations. As a matter of fact, studies in adult populations showed a negative correlation between plasma 
LTF levels and weight. LTF administration to Japanese adults led to decreased body weight and BMI (26) and, in male with altered glucose tolerance, plasma LTF concentration was inversely proportional with fasting TG, BMI, and waist-to-hip ratio, and was positively correlated with $\operatorname{HDL}-\mathrm{C}(12,27)$. Also in adults, LTF protein concentrations in visceral and subcutaneous adipose tissue were inversely associated with BMI and percent fat mass (28). To explain these differences between children and adults, Kim et al. (14) proposed that, in adults, hyperglycemia or inflammatory responses could interrupt degranulation of neutrophils and thus reduce the LTF secretion efficiency, a phenomenon that would not occur in children, given that their inflammation-related metabolic status may be less impacted in children .

Interestingly, in our study, after segregating according to HDL-C levels, the observed associations with weight and BMI remained significant only in children presenting with low HDL-C. Accordingly, LTF levels were reported to be elevated in inflammatory situations $(29,30)$ and LTF antiinflammatory activity is partially based on its ability to bind free ferric ions and inhibit oxidative bursts. Hence, the positive association between LTF levels and weight/BMI in children presenting with low HDL-C could reflect a compensatory mechanism in subjects prone to inflammation, given the powerful anti-inflammatory and anti-oxidant capacities attributed to HDL-C particles.

Furthermore, our study found a gender-dependant positive correlation between plasma LTF and lipid levels, namely total cholesterol, LDL-C and apo B, but the association was only significant in girls. Similar sex-specific associations were found with LDL-C in Latino girls, which were not significant in boys (14). This gender-dependent association was also found for markers of obesity (BMI, fat mass, waist, and hip circumferences) that accompanied higher LTF concentrations in boys alone (14). In addition, the associations reported in our investigation are different from the findings reported in adult cohorts, as LDL-C levels were found negatively (12) or not correlated (13) with LTF. Importantly, unlike our cohort, these studies were conducted on adult men only (12) and in severely obese subjects (13). The gender and age associations observed in our study are difficult to explain at the moment, but one could speculate that they might be related to the inflammatory and/or adiposity status. Nonetheless, they stress the need to further examine the effects of plasma LTF in younger populations, taking into account subjects' adiposity status.

In summary, the association between 13 LTF polymorphisms and cardio-metabolic risk factors was investigated in a French-Canadian pediatric population. Although these polymorphisms were not independent risk factors for various components of the metabolic syndrome, our findings emphasize a number of significant associations between HDL-C and gender on the one hand, and plasma LTF, anthropometric profile, and lipid levels on the other hand, which may contribute to the pathogenesis of the metabolic syndrome.

\section{SUPPLEMENTARY MATERIAL}

Supplementary material is linked to the online version of the paper at http://www.nature.com/pr

\section{ACKNOWLEDGMENTS}

We thank Schohraya Spahis for her technical assistance and acknowledge the assistance of Marie Lambert (deceased) for allowing us to access the QCAHSS cohort.

\section{STATEMENT OF FINANCIAL SUPPORT}

This work was supported by grants from the Dairy Farmers of Canada and J.A. de Sève Research Chair in Nutrition (E.L.), the Canadian Institutes of Health Research Fellowship Award and The Richard and Edith Strauss Postdoctoral Fellowships Award in Medicine, McGill University (V.M.).

Disclosure: The authors declare no conflict of interest.

\section{REFERENCES}

1. Alberti KG, Zimmet P, Shaw J. Metabolic syndrome-a new world-wide definition. A Consensus Statement from the International Diabetes Federation. Diabet Med 2006;23:469-80.

2. Eppens MC, Craig ME, Jones TW, Silink M, Ong S, Ping YJ. Type 2 diabetes in youth from the Western Pacific region: glycaemic control, diabetes care and complications. Curr Med Res Opin 2006;22:1013-20.

3. Suzuki YA, Lonnerdal B. Baculovirus expression of mouse lactoferrin receptor and tissue distribution in the mouse. Biometals 2004;17:301-9.

4. Farnaud S, Evans RW. Lactoferrin-a multifunctional protein with antimicrobial properties. Mol Immunol 2003;40:395-405.

5. Picard-Jean F, Bouchard S, Larivee G, Bisaillon M. The intracellular inhibition of $\mathrm{HCV}$ replication represents a novel mechanism of action by the innate immune lactoferrin protein. Antiviral Res 2014;111:13-22.

6. Cohen MS, Mao J, Rasmussen GT, Serody JS, Britigan BE. Interaction of lactoferrin and lipopolysaccharide (LPS): effects on the antioxidant property of lactoferrin and the ability of LPS to prime human neutrophils for enhanced superoxide formation. J Infect Dis 1992;166:1375-8.

7. Safaeian L, Zabolian H. Antioxidant effects of bovine lactoferrin on dexamethasone-induced hypertension in rat. ISRN Pharmacol 2014;2014: 943523.

8. Takeuchi T, Shimizu H, Ando K, Harada E. Bovine lactoferrin reduces plasma triacylglycerol and NEFA accompanied by decreased hepatic cholesterol and triacylglycerol contents in rodents. Br J Nutr 2004;91: 533-8.

9. Morishita S, Ono T, Fujisaki C, et al. Bovine lactoferrin reduces visceral fat and liver triglycerides in ICR mice. J Oleo Sci 2013;62:97-103.

10. Kajikawa M, Ohta T, Takase M, Kawase K, Shimamura S, Matsuda I. Lactoferrin inhibits cholesterol accumulation in macrophages mediated by acetylated or oxidized low-density lipoproteins. Biochim Biophys Acta 1994;1213:82-90.

11. Suginohara Y, Miyazaki A, Hakamata H, et al. The heparin-bound fraction of human lipoprotein-deficient serum inhibits endocytic uptake of oxidized low density lipoprotein by macrophages. Atherosclerosis 1996;120:167-79.

12. Moreno-Navarrete JM, Ortega FJ, Bassols J, Castro A, Ricart W, Fernandez-Real JM. Association of circulating lactoferrin concentration and 2 nonsynonymous LTF gene polymorphisms with dyslipidemia in men depends on glucose-tolerance status. Clin Chem 2008;54:301-9.

13. Fernandez-Real JM, Garcia-Fuentes E, Moreno-Navarrete JM, et al. Fat overload induces changes in circulating lactoferrin that are associated with postprandial lipemia and oxidative stress in severely obese subjects. Obesity 2010;18:482-8.

14. Kim JY, Campbell LE, Shaibi GQ, Coletta DK. Gene expression profiling and association of circulating lactoferrin level with obesity-related phenotypes in Latino youth. Pediatr Obes 2014;10:338-44.

15. Videm V, Dahl H, Walberg LE, Wiseth R. Functional polymorphisms in the LTF gene and risk of coronary artery stenosis. Hum Immunol 2012;73:554-9. 


\section{Articles | Marcil et al.}

16. Mohamed JA, DuPont HL, Jiang ZD, et al. A novel single-nucleotide polymorphism in the lactoferrin gene is associated with susceptibility to diarrhea in North American travelers to Mexico. Clin Infect Dis 2007;44:945-52.

17. Borie C, Gasparini F, Verpillat P, et al. Association study between ironrelated genes polymorphisms and Parkinson's disease. J Neurol 2002;249: 801-4.

18. Paradis G, Lambert M, O'Loughlin J, et al. The Quebec Child and Adolescent Health and Social Survey: design and methods of a cardiovascular risk factor survey for youth. Can J Cardiol 2003;19:523-31.

19. Marcil V, Amre D, Seidman EG, et al. Hepatocyte nuclear factor 4 alpha polymorphisms and the metabolic syndrome in French-Canadian youth. PLoS ONE 2015;10:e0117238.

20. Araki-Sasaki K, Ando Y, Nakamura M, et al. Lactoferrin Glu561Asp facilitates secondary amyloidosis in the cornea. Br J Ophthalmol 2005;89: 684-8.

21. Azevedo LF, Pecharki GD, Brancher JA, et al. Analysis of the association between lactotransferrin (LTF) gene polymorphism and dental caries. J Appl Oral Sci 2010;18:166-70.

22. Jordan WJ, Eskdale J, Lennon GP, et al. A non-conservative, coding single-nucleotide polymorphism in the $\mathrm{N}$-terminal region of lactoferrin is associated with aggressive periodontitis in an African-American, but not a Caucasian population. Genes Immun 2005;6:632-5.

23. Kachiwala SJ, Harris SE, Wright AF, et al. Genetic influences on oxidative stress and their association with normal cognitive ageing. Neurosci Lett 2005;386:116-20.
24. Keijser S, Jager MJ, Dogterom-Ballering HC, et al. Lactoferrin Glu561Asp polymorphism is associated with susceptibility to herpes simplex keratitis. Exp Eye Res 2008;86:105-9.

25. Wu YM, Juo SH, Ho YP, Ho KY, Yang YH, Tsai CC. Association between lactoferrin gene polymorphisms and aggressive periodontitis among Taiwanese patients. J Periodontal Res 2009;44:418-24.

26. Ono T, Murakoshi M, Suzuki N, et al. Potent anti-obesity effect of enteric-coated lactoferrin: decrease in visceral fat accumulation in Japanese men and women with abdominal obesity after 8-week administration of enteric-coated lactoferrin tablets. Br J Nutr 2010;104: 1688-95.

27. Moreno-Navarrete JM, Ortega FJ, Bassols J, Ricart W, Fernandez-Real JM. Decreased circulating lactoferrin in insulin resistance and altered glucose tolerance as a possible marker of neutrophil dysfunction in type 2 diabetes. J Clin Endocrinol Metab 2009;94:4036-4.

28. Moreno-Navarrete JM, Serrano M, Sabater M, et al. Study of lactoferrin gene expression in human and mouse adipose tissue, human preadipocytes and mouse 3T3-L1 fibroblasts. Association with adipogenic and inflammatory markers. J Nutr Biochem 2013;24:1266-75.

29. Berkestedt I, Herwald H, Ljunggren L, Nelson A, Bodelsson M. Elevated plasma levels of antimicrobial polypeptides in patients with severe sepsis. J. Innate Immun 2010;2:478-82.

30. Miyauchi S, Umekita K, Hidaka T, et al. Increased plasma lactoferrin levels in leucocytapheresis therapy in patients with rheumatoid arthritis. Rheumatology 2014;53:1966-72. 